\title{
The use of COPS and NSIS in the EuQoS Project
}

\author{
Edmundo Monteiro, Fernando Boavida, Paulo Simões, Jorge Sá Silva, \\ Marilia Curado, Luís Cordeiro, Romulo Ribeiro, Maxweel Carmo, Jian \\ Zhang \\ University of Coimbra \\ Laboratory of Communications and Telematics \\ CISUC-DEI \\ Pólo II, 3030-290 Coimbra - Portugal \\ \{edmundo,boavida,psimoes,sasilva,marilia,cordeiro,romulo,maxweel\} \\ @dei.uc.pt
}

\begin{abstract}
This paper discusses the use of the Policy Based Network paradigm, COPS (Common Open Policy Service) and NSIS (Next Steps In Signalling) frameworks being developed by the IETF, in the context of the European Project EuQoS (End-to-End Quality of Service over Heterogeneous Networks). An overview of the EuQoS architecture is provided followed by the analysis of the use of PBN/COPS and NSIS to support the Signalling and Service Negotiation (SSN) function. The implementation status and validation aspects are also discussed in the paper and some preliminary results are included.
\end{abstract}

\section{Introduction}

The motivation of the European Integrated Project EuQoS (End-to-end Quality of Service support over heterogeneous networks) [1] is to solve the outstanding design issues presently associated with the delivery of end to end QoS service across heterogeneous networks. It is necessary to resolve these issues and accordingly upgrade the infrastructures so that new applications can be supported by the Internet and new service packages can be offered by operators and service providers.

The key objective of EuQoS is to research, integrate, test, validate and demonstrate end-to-end QoS technologies to support the infrastructure upgrade for advanced QoS-aware applications over multiple, heterogeneous network domains, belonging to research, scientific and industrial communities. The project will deliver the EuQoS system which will support the delivery of end to end QoS. As QoS is primarily a challenge for the access network, the EuQoS system will be developed and tested on various types of research access networks with the GEANT [2] core providing Pan European support. This heterogeneous infrastructure, which models 
future production networks, requires a QoS technical solution that has not been synthesized to date.

The EuQoS project will propose and develop new QoS mechanisms build upon the state of the art and incorporate the following components: Monitoring and Measurement, Admission Control, Failure Management, Signalling \& Service Negotiation, Security and AAA, Charging and Traffic Engineering and Resource Optimization. EuQoS will integrate state of the art protocols and technologies with new mechanisms to be developed in the project in order to build the above specified functions.

This paper addresses the use of the Policy Based Network (PBN) paradigm [3] and its support protocol, the Common Open Policy Service (COPS) [4] together with the NSIS (Next Steps in Signalling) IETF (Internet Engineering Task Force) framework [5] in order to support some of the EuQoS functionalities.

The remaining of the paper is structured as follows. The EuQoS architecture is briefly described in Section 2. Sections 3 and 4 provide some background about PBN and NSIS. Section 5 describes the proposed solution. Validation issues are discussed in Section 6. Finally, Section 7 concludes the paper and points out some directions for future work in the scope of the EuQoS project.

\section{EuQoS Architecture}

The EuQoS project aims at creating a flexible and secure QoS Assurance System (the EuQoS System) by developing new QoS mechanisms which build upon the state-of-the-art. The EuQoS System consists of two major research components: User and QoS aware Control Plane and QoS Protocol Stack, as shown in Figure 1. The QoS Protocol Stack will result in a new API over existing and new transport protocols that will provide variable levels of order and reliability.

The Control Plane will include a set of functions that might be supported in network elements such as routers and in end systems. To integrate the Control Plane of the EuQoS architecture six main functions were identified: Function 1 - Signalling and Service Negotiation (SSN); Function 2 - Connection Admission Control (CAC); Function 3 - Monitoring Measurement, Fault Management (MMFM); Function 4 Traffic Engineering and Resource Optimization (TERO); Function 5 - Security and AAA (SAAA); Function 6- Charging (CHAR).

The SSN function is responsible for the dynamic configuration of the communication system, in order to provide the requested level of QoS to applications. It covers the application connection setup, data transfer and teardown phases and is triggered by applications located at end systems, by application proxies or by network elements.

The EuQoS SSN function will be built over state-of-art signalling and service negotiation mechanisms complemented with new functionalities to be researched and developed in the EuQoS project. Among other proposals the PBN, COPS and NSIS will play an important role in EuQoS as will be described in the following sections. 


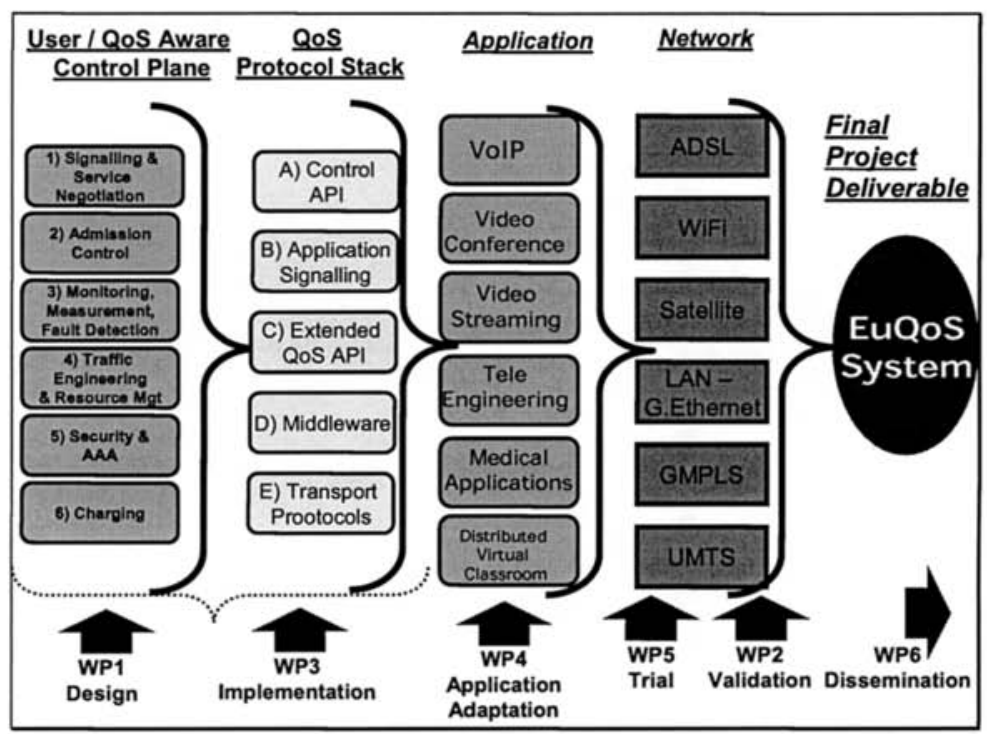

Fig. 1. The EuQoS system

\section{PBN and COPS in EuQoS architecture}

This section discusses the use of the Policy-Based Networking and the Common Open Policy Service in the EuQoS project context.

\subsection{Policy-Based Networking Concepts}

The PBN concept and architecture were developed by IETF's Policy Framework group [3]. The objective of PBN is to enable centralized network administration by supporting the definition of abstract rules, that is, by specifying what the network should do in a high-level language instead of specifying how to do it. This is done in a network-element-independent way, through a policy specification language.

The PBN architecture comprises four basic components: Management Console, Policy Repository (PR), Policy Decision Point (PDP) and Policy Enforcement Point (PEP). The Management Console provides an interface between the administrator and the policy management system. The PR stores the policies that are applicable to a given administration. Every policy database is structured as a Policy Information Base (PIB). PDPs translate high-level policies stored in the PR into lower level policy rules. PEPs receive rules from PDPs and deal with network elements accordingly, performing actions such as packet filtering and marking. The communication between PDPs and PEPs is achieved using a policy transfer protocol such as COPS protocol [4] or COPS-PR (COPS for Policy Provisioning) [6]. 


\subsection{PBN in the Context of EuQoS}

PBN plays an important role in the EuQoS system, since it provides an elegant and technology independent schema to map high-level QoS domain policies into lowlevel network equipment configuration, coping with the required autonomy of QoSdomain management boundaries and the need to establish a network technology independent sub-layer that aggregates the Resource Managers (RM) of the various QoS domains.

Figure 2 shows how COPS fits into the EuQoS architecture. Each administrative domain (QoS Domain) maintains its own Policy Repository and Policy Decision Point. The PR stores domain-specific policies according to an LDAP (Lightweight Directory Access Protocol) schema. The policies are then used at two distinct levels:

- To define the technology-independent behaviour of the Resource Manager (which QoS requests should be satisfied, under what circumstances);

- To translate QoS requirements into specific network equipment configuration, using COPS-PR for the communication between the PDP and the PEPs at the managed network nodes.

In this way, PBN plays an important role in the definition of the QoS Domain as a whole (policies regarding interaction with peer QoS domains) and in the translation of high-level policies into equipment specific configurations.
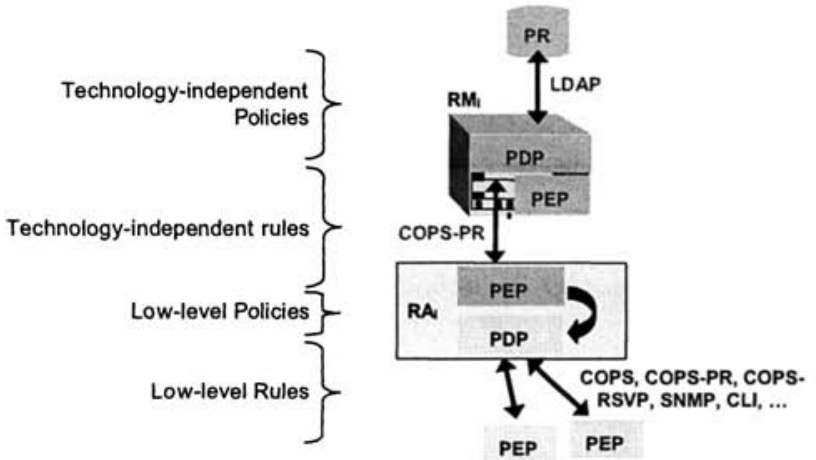

Fig. 2. $P B N$ in the EuQoS context

\section{NSIS in the EuQoS Context}

This section discusses the use of the NSIS framework in the EuQoS project.

\subsection{NSIS Concepts}

The Next Steps in Signalling suite of protocols is being developed by the IETF NSIS working group [5]. The NSIS framework aims at providing signalling elements for the global Internet environment, to answer different needs (e.g. resource reservation, firewall transversal), without requiring a complete end-to-end deployment. Signal- 
ling protocol messages can take either the same path as the data path between endpoints or a different path (on-path and off-path signalling).

The NSIS protocol stack is divided in two layers: a generic lower layer for signalling transportation and an upper layer specific to each signalling application. For the lower layer a General Internet Messaging Protocol for Signalling (GIMPS) [7] is currently being standardized. For the upper layer the NSIS working group is currently working in two protocols: the QoS NSIS Signalling Layer Protocol (QoSNSLP) [8] for resource reservation and QoS support and the NAT/Firewall NSIS Signalling Layer Protocol (NAT/FW-NSLP) for firewall transversal [9].

\subsection{NSIS in the context of EuQoS}

The NSIS framework is well fitted for the EuQoS project architecture. GIMPS provides the signalling transport mechanism that can be used between RMs (off-path signalling) and also between routers and other network equipment (on-path signalling). This protocol also provides an abstraction layer that can be used for all the high-level signalling functions needed in the EuQoS framework. QoS-NSLP provides mechanisms to establish and maintain state at nodes along the path of a data flow in an end-to-end environment and can also be used for resource reservation between RMs, across network domains.

The main limitation for the adoption of the complete NSIS framework in the EuQoS context is its relative immaturity, affecting mainly the application signalling protocols like QoS-NSLP. To overcome this limitation, the use of NSIS in EuQoS started by GIMPS and will progressively include QoS-NSLP.

\section{Putting it all together: the SSN Function}

The Signalling and Service Negotiation function is responsible for the dynamic configuration of the communication system, in order to provide the requested level of QoS to applications.

\subsection{General Overview of the SSN Function}

There are four different "signalling levels" in the EuQoS architecture, as illustrated in Figure 3 by the various red arrows (Level 1 to Level 4).

Besides horizontal signalling interactions between peer entities, there are complementary signalling interactions that take place vertically between adjacent levels. These interactions are named "cross-layer signalling" and are identified by the vertical arrows in Figure 3. 


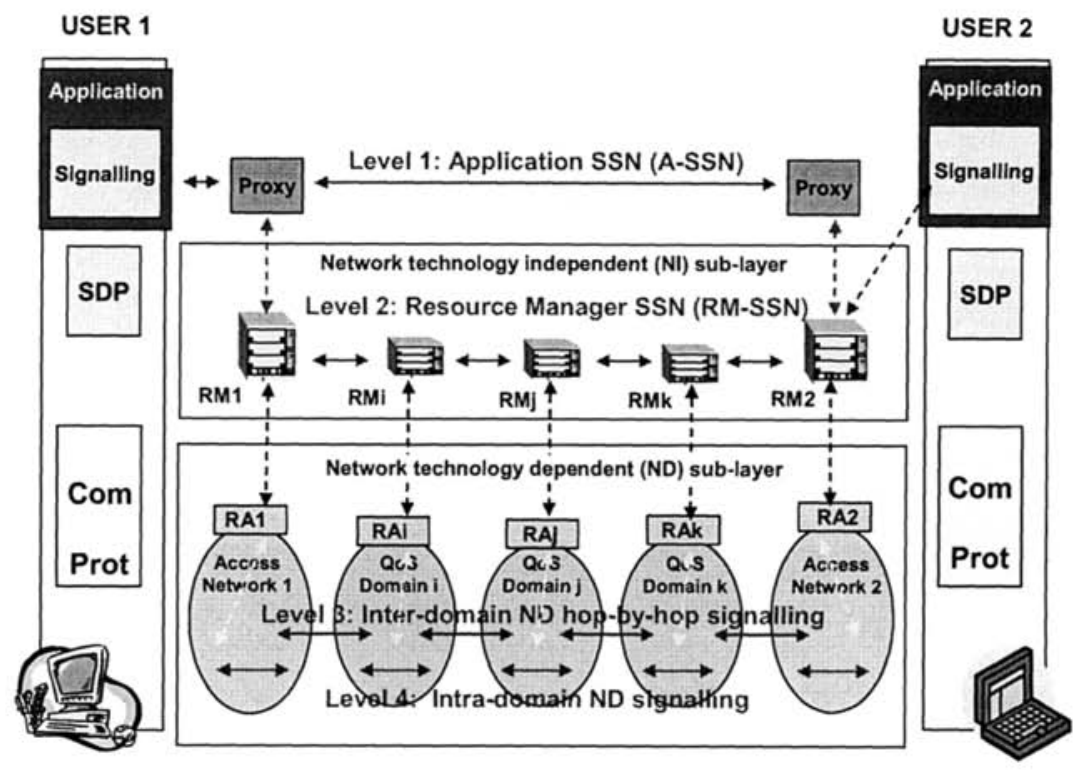

Fig. 3. Signalling levels in the EuQoS architecture

\subsection{Level 1: A-SSN}

The first signalling level pertains to applications and provides Application QoSbased Signalling and Service Negotiation (A-SSN). In order to establish, maintain and release sessions with the necessary QoS levels, applications must express their needs and interact with the communication system. The objectives of this interaction are to guarantee that the needs of the application will be fulfilled, and that it will adapt to network conditions under which the available resources don't allow the requested level of QoS. Furthermore, the interaction between applications and the communication system will be responsible for releasing the resources when sessions end. The main requirements of Level 1 signalling functions are:

- Identification of users, accounting and billing functions (AAA functions);

- Negotiation and definition of session characteristics between user terminals including the possibility of choice of QoS characteristics by the user;

- Prioritization of data-flows;

- Identification of QoS requirements for the communication system;

- Verification of the possibility to setup connections with the required (or available) quality requirements (by interacting with $\mathrm{CAC}$ functions);

- Set-up connections between user terminals with the required (or available) quality level (by interacting with RMs);

- Maintain the quality level during sessions or adapt to quality variation in the communication system (by interacting with RMs);

- Release communication system resources at the end of sessions;

- Provide information to users about the quality level and relevant session characteristics during session set-up, session life and at session tear-down. 
Signalling interactions between applications and the communication system can be explicitly made in the control plane by using a signalling protocol like SIP [11], or they can be implicitly initiated by the inspection of the data path and detection of session activation and termination. When explicit signalling is used, Level 1 signalling can be direct end-to-end between the applications or mediated by proxy entities. If implicit signalling is used, the quality requirements of applications have to be defined a priori by some sort of mechanism (e.g. policy mechanisms defined at application and/or user level). The interaction between Level 1 signalling functions and RMs is achieved by the vertical interactions as depicted by the vertical arrows in Figure 3.

For the purpose of EuQoS, SIP QoS extensions will be used and named in the context of this document as EQ-SIP. The vertical interactions between RMs and RAs, and between RAs and network equipment can be supported, among others solutions, by the COPS protocol or by SNMP.

\subsection{Level 2: RM-SSN}

The Resource Managers Signalling and Service Negotiation (RM-SSN) is the most important and the most complex to be developed in the EuQoS architecture. The main objective of Level 2 signalling functions is to support resource reservation and management along the data path across the various network domains. The main requirements of Level 2 functions are:

- Activation of SLSs with adjacent domains (chosen by the TERO function) in reaction to local application needs (triggered by Level 1 signalling);

- Activation, renegotiation, reception and termination of SLSs with adjacent domains, in reaction to local application needs (triggered by Level 1 signalling) and quality level variations;

- Verification of the availability of resources to support the requested SLAs with the specified (or available) quality requirements (by interacting with CAC functions);

- Maintenance of SLSs with adjacent domains with the specified quality (and renegotiation of the SLAs when needed);

- Support SLS Monitoring and Measurement (interface with MMFM function), and support SLS optimization (interface with TERO function).

- To support inter-domain service negotiation, Level 2 signalling functions will perform hop-by-hop negotiation between RMs in the path starting in the local RM at the origin access network (Access Network 1 domain in Figure 3). Each RM interacts with the Traffic Engineering and Resource Optimization (TERO) function to find the next hop domain, install provisional reservations if resources are available (using the CAC function that, in turn, will use cross-layer signalling identified by the blue arrow in Figure 3 to interact with the RA of the domain), and then will start signalling with the next domain's RM, which will repeat the process until the last RM in the path is reached (Access Network 2 domain in Figure 3). When the remote RM is reached, signalling is sent in the opposite direction to confirm the provisional reservations made in the downstream direction. Alternatively to the use of the TERO function to find next hops between domains, this can be done in a static way at each RM. The options to support Level 2 signalling include the development of a specific solution for the EuQoS architecture, the use 
of the SLS negotiation mechanism developed in the scope of the European IST project Mescal [12], the use of the COPS extension for SLS negotiation (COPSSLS) [13], or the use of NSIS. Figure 4 shows the utilization of a simplified version of NSIS in the EuQoS project, named EQ-NSIS.

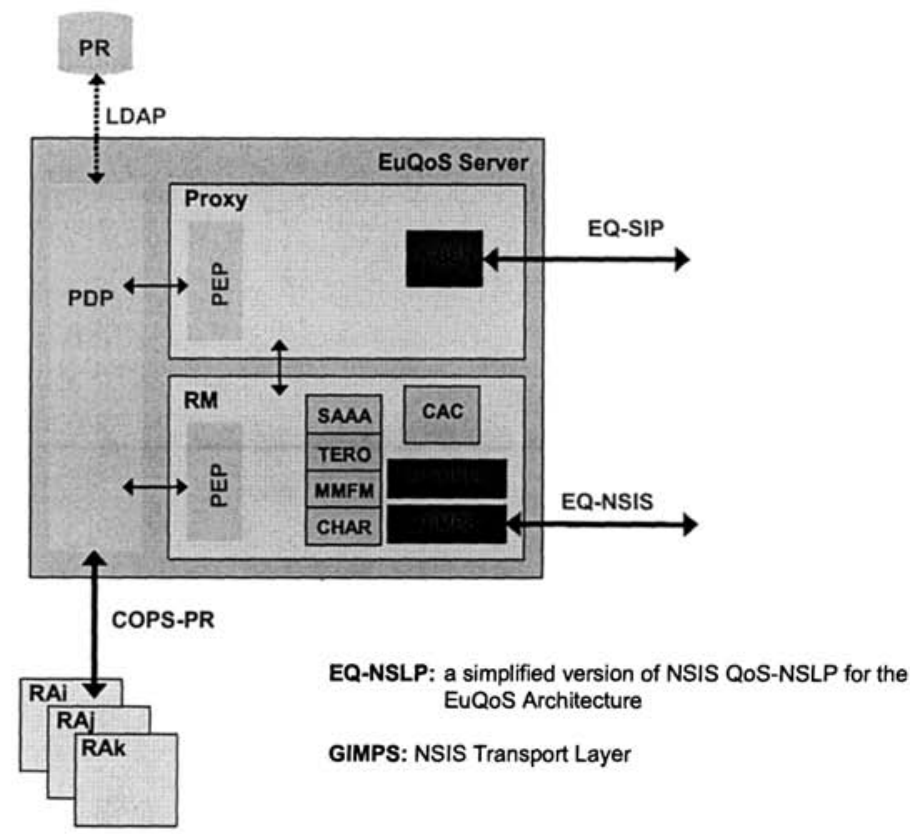

Fig. 4. EuQoS signalling at application and RM levels

\subsection{Level 3: Inter-domain signalling}

The third level of signalling in the EuQoS SSN Function is the network-technologydependent hop-by-hop inter-domain signalling. At this level BGP (Border Gateway Protocol) is the most common solution currently used. BGP provides mechanisms for inter-domain traffic routing and enables the use of routing policies to control the exchange of routing information between different administrative domains (Autonomous Systems).

The BGP version currently in use supports only Best Effort traffic, but extensions, usually called BGP+, are being studied to deal with different traffic classes with different QoS requirements. The use of BGP+ enable high level RMs to rely on network level mechanisms for QoS support and network resilience.

The control of BGP+ routing policies can be made by RAs (and, indirectly, by RMs using cross-layer signalling interactions) to route specific traffic classes via specific intermediate domains according to QoS and business requirements. Traffic Engineering and Resource Optimization (TERO) and Monitoring Measurement, Fault Management (MMFM) functions can provide useful information about the state of the network to support the policy control decisions. Figure 5 illustrates the 
use of BGP+ inter-domain signalling, as well as intra-domain signalling interactions (discussed below).

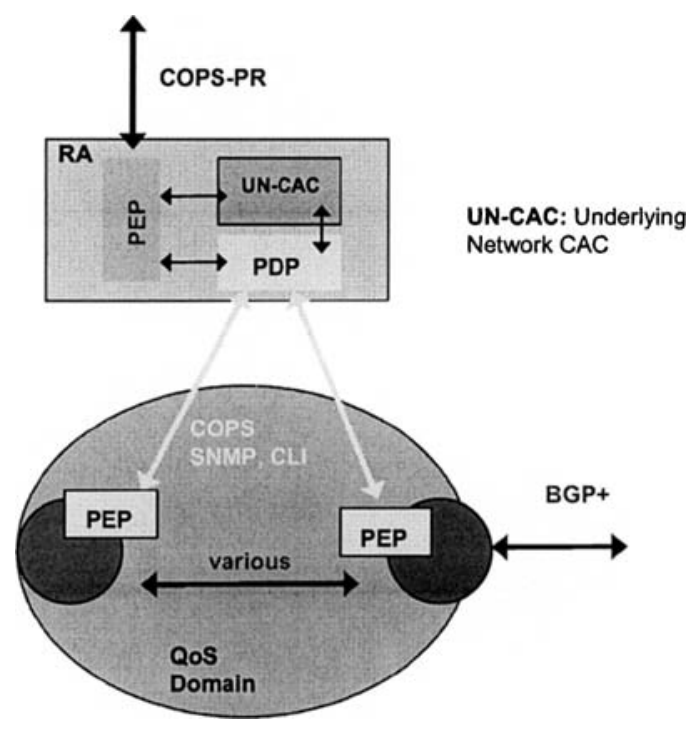

Fig. 5. Inter-domain ND hop-by-hop signalling in the EuQoS architecture

\subsection{Level 4: Intra-domain signalling}

The configuration of the resources of a domain is performed by RAs using solutions ranging from RSVP to specific access network mechanism like ADSL bandwidth management, $3 \mathrm{GPP}$, and Ethernet and WIFI priorities. The path-coupled version of NSIS can also be used for this level of signalling. When these signalling mechanisms are not available, the RAs must have a detailed knowledge of the domain configuration and topology so they can act directly in the configuration of each network element along the path subject to QoS requirements, using cross-layer signalling interactions.

Figure 6 presents the overall EuQoS signalling picture, according to the description presented above. 


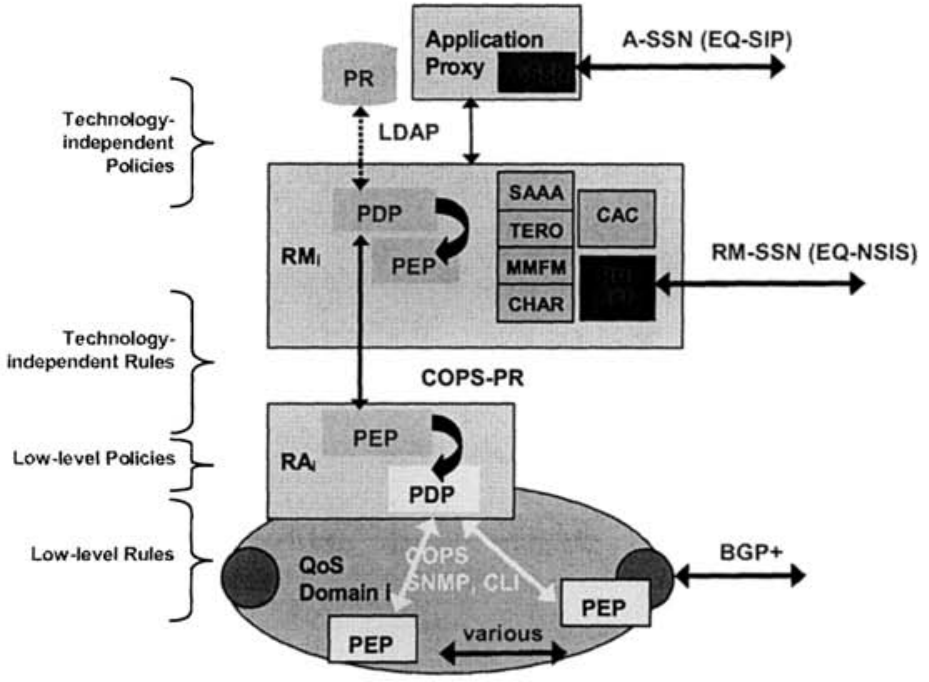

Fig. 6. Overall in-layer and cross-layer signalling protocols in the EuQoS architecture

\section{Validation}

The EuQoS system will be validated by simulation, in the NS-2 framework, and by trial evaluation in wide test scenarios including different user communities, applications and access technologies. The main objective of the experiments is to evaluate its capacity for delivering end-to-end QoS with special emphasis on the QoS mechanisms and algorithms developed in the scope of the project.

\subsection{Simulation}

In this section we present one of the studies that we made to evaluate the NSIS advantages in network scenarios, particularly by the use of the GIMPS layer specified in the NSIS framework, when it is necessary to implement a QoS signalling mechanism. For this purpose, we have developed a set of new modules for NS-2: $802.1 \mathrm{p} / \mathrm{Q}$ and a NSIS package.

A simple NSLP has also been deployed to work on an Ethernet switch. Our NSLP interacts with the switch in order to configure prioritization levels, using the IEEE $802.1 \mathrm{p}$ and $\mathrm{Q}$ standards. The next figure presents the Small Office Home Office (SOHO) scenario used in the study.

For foreground traffic we decided to use VoIP G.711 sources without Voice Activity Detection (VAD), as described in Table 1. 


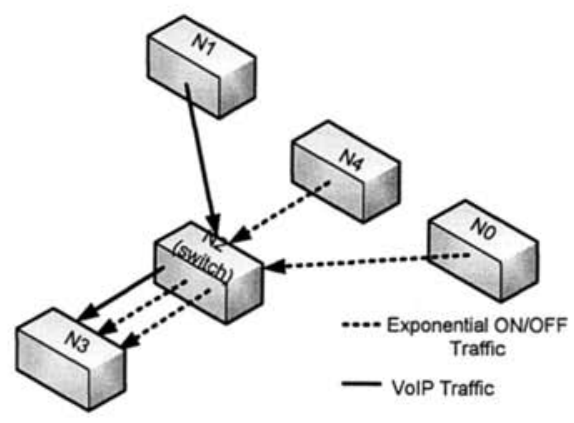

Fig. 7. Scenario \#1

Table 1. Source parameters

\begin{tabular}{llll}
\hline Traffic Type & VoIP & Traffic1 & Traffic1 \\
\hline Description & G711 without VAD & Packet Size 800 & PacketSize 200 \\
& Packet size 200 B & Burst_time 0.03 & Burst_time 0.02 \\
& Inter-arrival 0.02 s & Idle_time 0.03 & Idle_time 0.02 \\
& & Rate 1000k & Rate 600k \\
& & Exponential traffic & Exponential traffic \\
\hline
\end{tabular}

The purpose of this study was to evaluate the NSIS effect on the traffic and on the congestion level. In these simulations, the traffic between the nodes N1 and N3 was modelled as G.711 VoIP, requiring a high prioritization level. The background traffic (from N0 and N4) was modelled as exponential ON/OFF sources in order to overload the links. A NSIS agent was configured in each node.

Figure 8 shows the one way delay (OWD) experienced by each of the traffic flows, when no prioritization was performed in the switch. In this case, the switch had just two queues. As expected, all the traffic presented similar behaviour, experiencing high delays in the face of network congestion.

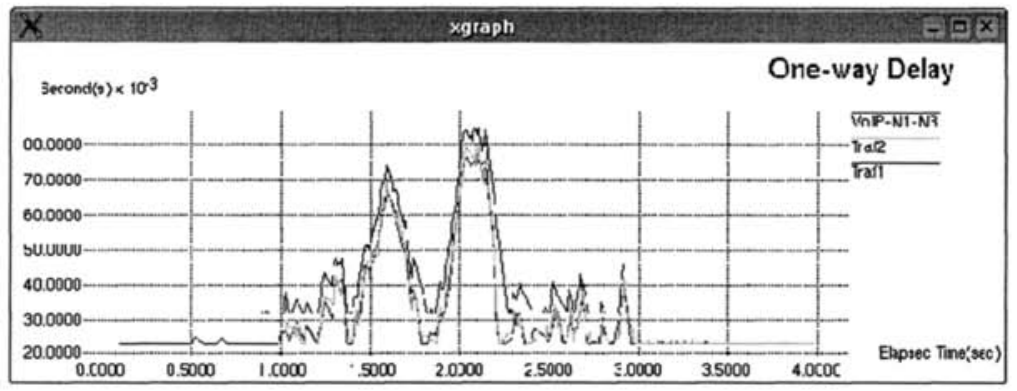

Fig. 8. Packet delay without traffic prioritization

Figure 9 shows the OWD when prioritization was introduced in the switch, activated by a NSIS signalling message. The switch was initially configured with 2 
queues, and no prioritization mechanism was used. At $1.3 \mathrm{sec}$, the NSIS agent, located in N1, sent a path-message to node N3. The payload of this NSIS message contained the command to reconfigure the switch $(\mathrm{N} 1 \Rightarrow \mathrm{N} 2 \Rightarrow \mathrm{N} 3)$ with 7 virtual queues. Each NSIS agent initialized the peer discovery negotiations while the NSLP function reconfigured each switch along the data path. After that, traffic was sent between $\mathrm{N} 1$ and N3. As can be observed in figure 9, the VoIP delay remained low during all the simulation.

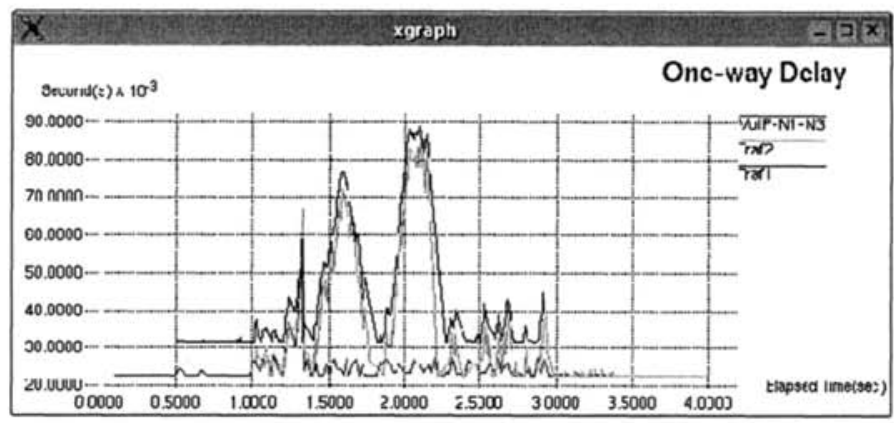

Fig. 9. Packet delay with traffic prioritization after the NSIS Message

These simulation tests demonstrated NSIS advantages when it is necessary to implement different classes of traffic. Different NSIS mechanisms can be built, extending the NSLP functionalities used in this example.

\section{Conclusion}

This paper discussed the use of the Policy Based Network paradigm, COPS and NSIS frameworks being developed by IETF, in the context of the European Project EuQoS. The analysis of the use of PBN/COPS and NSIS to support the SSN function revealed that these protocols can be used to provide a basis to support the desired functionality and a framework for further enhancements.

The implementation is being carried out in two parallel tracks: simulations models and prototype that will support the validation tasks planned in the EuQoS project. Preliminary simulation results show that NSIS has potential to control underlying network devices and improve the QoS provided to applications.

\section{Acknowledgements}

This work was partially funded by the European Union $6^{\text {th }}$ Framework Programme under contract IST FP6 IP 004503 EuQoS Integrated Project. The authors also acknowledge the various comments and valuable suggestions received from the EuQoS project team. 


\section{References}

1. EuQoS Project consortium, Integrated Project EuQoS End-to-end Quality of Service support over heterogeneous networks Annex I - DoW, May 2004.

2. The GÉANT project - http://www.geant.net

3. IETF Policy Framework Working Group (IETF-PG) http://www.ietf.org/html.charters/policy-charter.html

4. Durham, Ed., J. Boyle, R. Cohen, S. Herzog, R. Rajan, A. Sastry, The COPS (Common Open Policy Service) Protocol, RFC 2748, IETF, January 2000.

5. R. Hancock G. Karagiannis J. Loughney S. van den Bosch, Next Steps in Signaling: Framework, Internet-Draft, IETF, 2005.

6. K. Chan, J. Seligson, D. Durham, S. Gai, K. McCloghrie, S. Herzog, F. Reichmeyer, R. Yavatkar, A. Smith, COPS Usage for Policy Provisioning (COPS-PR), RFC 3084, IETF, March 2001.

7. Schulzrinne, R. Hancock, GIMPS: General Internet Messaging Protocol for Signaling, Internet-Draft, IETF, 2004.

8. S. Van den Bosch, G. Karagiannis, A. McDonald, NSLP for Quality-of-Service signaling, Internet-Draft, IETF, 2004.

9. M. Stiemerling H. Tschofenig M. Martin, C. Aoun, NAT/Firewall NSIS Signaling Layer Protocol (NSLP), Internet-Draft, IETF, 2004.

10. R. Braden, L. Zhang, S. Berson, S. Herzog, S. Jamin, Resource ReSerVation Protocol (RSVP), RFC 2205, IETF, September 1997.

11. Handley, H. Schulzrinne, E. Schooler, J. Rosenberg, SIP: Session Initiation Protocol, MRFC 2543, March 1999

12. MESCAL Project, Management of End-to-end Quality of Service Across the Internet at Large - http://www.mescal.org/

13. T.M.T. Nguyen, G. Pujolle, N. Boukhatem, COPS Usage for SLS negotiation (COPS-SLS), Internet-Draft, IETF, June 2001. 